\title{
Effects of Surface Diffuseness and Coupling Radius Parameters on The Fusion and Quasi-Elastic Barrier Distributions
}

\author{
Viska Inda Variani, Ida Usman, and Muhammad Zamrun Firihu \\ Jurusan Fisika, Fakultas MIPA, Universitas Halu Oleo, Kendari, Sulwesi Tenggara, 93232 \\ mzamrun@uho.ac.id
}

\begin{abstract}
We study the heavy-ion reaction at sub-barrier energies for ${ }^{16} \mathrm{O}+{ }^{144,154} \mathrm{~S} \mathrm{~m}$ systems using full order coupled-channels formalism. We especially investigate the effect of fusion and quasielastic barrier distributions on the surface diffuseness and the coupling radius parameters of the nuclear potential for these systems. We found that the structure of fusion and quasi-elastic barrier distributions is more sensitive to the surface diffuseness and coupling radius parameters for the reaction with spherical target, ${ }^{16} \mathrm{O}+{ }^{144} \mathrm{Sm}$ systemcompared to the reaction that involves the deformed target, i.e., ${ }^{16} \mathrm{O}+{ }^{154} \mathrm{Sm}$ system. In more detail, the results of coupled-channels calculations for the fusion and the quasi-elastic barrier distributions for deformed target are not sensitive to the choice of the coupling radius and surface diffuseness parameters. In mark contrast, the structure of the fusion and the quasi-elastic barrier distributions for spherical target are very sensitive to the coupling radius and surface diffuseness parameters. We found that the small surface diffuseness parameter smeared out the fusion barrier distributions and the larger coupling radius smoothed the high energy peak of the quasi-elastic barrier distributions. We also found that the larger coupling radius, $r_{\text {coup }}=1.20 \mathrm{fm}$, is required by the experimental quasi-elastic barrier distribution for the ${ }^{16} \mathrm{O}+{ }^{144} \mathrm{Sm}$ system whereas the experimental fusion barrier distribution compulsory the small value, i.e., $r_{\text {coup }}=1.06 \mathrm{fm}$.
\end{abstract}

Keywords: heavy-ion reaction, coupled-channels, fusion, quasi-elastic, barrier distribution.

\section{INTRODUCTION}

It is now well established that the coupled-channels calculations for the heavy-ion reaction at energies near and below the Coulomb barrier can explained the experimental data of fusion reactions as well as the quasi-elastic scattering of the medium-heavy mass systems, heavy mass systems and a very heavy mass systems ${ }^{[1-12]}$. It is revealed that the cross section and the barrier distribution for fusion reactions and quasi-elastic scattering are very sensitive to the structure of the colliding nuclei, i.e., the collective excitations (rotations and surface vibrations), neck formation and/or nucleon transfer. Those effects can be more easily visualized through the so-called barrier distribution concept ${ }^{[13-15]}$.

Rowley et al. have proposed a method for extracting the fusion barrier distribution directly from experimental data of fusion cross sections, $\sigma_{f u s}(E)$, by taking the second derivative of the product $E \sigma_{f u s}(E)$ with respect to the center-of-mass energy, E, that is, $D^{f u s}=d^{2}\left(\sigma_{f u s}\right) /$ $d E^{2[13]}$. This concept can also be extended to the quasi-elastic scattering since the fusion reaction and the quasi-elastic scattering is related to each other because of the flux conservation ${ }^{[16]}$. In this case, the quasi-elastic barrier distribution is defined as the first derivative of the ratio of quasi-elastic to the Rutherford cross sections, $d \sigma_{q e l} / d \sigma_{R}$, with respect to energy $\mathrm{E}$, i.e. $D^{q e l}=-d\left(d \sigma_{q e l} / d \sigma_{R}\right) / d E^{[14,15]}$. It is well accepted that the 
concept of barrier distribution provides a powerful tool for investigating the effects of channel coupling on heavy-ion fusion reactions as well as quasi-elastic scattering at sub- barrier energies even for very heavy mass systems ${ }^{[7,9,17-21]}$.

In the coupled-channels analysis of heavy-ion reactions around the Coulomb barrier, the phenomenological Woods-Saxon potential for the nuclear part of the interaction between two heavy ions is widely used. Its parameters are usually adjusted in order to fit the experimental data. It is found that a much larger value of the surface diffuseness parameter, a, ranging from 0.75 to $1.5 \mathrm{fm}$, is required to fit the data ${ }^{[10,22-26]}$. In marked contrast, the value of the surface diffuseness parameter, $a=0.63 \mathrm{fm}$ is required by the scattering processes ${ }^{[27-32]}$. In the analysis of the experimental data with copuled-channels method the coupling radius parameter for the coupling potential, the value of $r_{\text {coup }}=1.06 \mathrm{fm}$ is often used. However, Ref. [7,9,27] the $r_{\text {coup }}=1.20 \mathrm{fm}$ is for explaining the experimental data of quasi-elastic scattering in massive systems ${ }^{[7,9,27]}$. The reason for the discrepancies in the diffuseness parameters extracted from scattering and fusion analysis has not yet been fully understood. And also, the difference coupling radius parameter used in the coupled- channels calculations have to be clarified.

In this paper, we carry out detailed full order coupled-channels calculations for the fu-sion reaction and large angle quasi-elastic scattering of the ${ }^{16} \mathrm{O}+{ }^{144,154} \mathrm{Sm}$ reactions. We especially discuss the sensitivity of fusion and quasi-elastic barrier distributions of these systems on the surface diffuseness parameter and the coupling radius parameter of the nuclear potential. The pioneer of this work has been done in Ref. [12].

The paper is organized as follows. We briefly explain the coupled-channels formalism for fusion reaction and large angle quasi-elastic scattering in Sec. II. The results of coupled- channels calculations are given in Sec. III. We summarize the paper in Sec. IV.

\section{COUPLED-CHANNELS FORMALISM FOR HEAVY-ION FUSION REACTION AND LARGE ANGLE QUASI-ELASTIC SCATTERING}

In this section, we briefly describe the coupled-channels formalism for fusion reaction and largeangle quasi-elastic scattering that includes the effects of the intrinsic excitations of the target nucleus. The total Hamiltonian of the system is assumed to be

$$
H=-\frac{\hbar^{2}}{2 \mu} \nabla^{2}+V_{N}^{(0)}(r)+\frac{Z_{P} Z_{T} e^{2}}{r}+H_{\text {exct }}+V_{\text {coup }}\left(\boldsymbol{r}, \xi_{T}\right),
$$

where $\mathbf{r}$ is the coordinate of the relative motion between the target and the projectile nuclei, $\mu$ is the reduced mass, and $\xi_{T}$ represents the coordinate of the intrinsic motion of the target nucleus. $Z_{P}$ and $Z_{T}$ are the atomic number of the projectile and the target, respectively, and $V_{N}^{(0)}(r)$ is the bare nuclear potential, which we assume to have a Woods-Saxon shape. It consists of the real and imaginary parts, $V_{N}^{(0)}(r)=V_{0}(r)+i W_{0}(r) . H_{\text {exct }}$ describes the excitation spectra of the target nucleus, whereas $V_{\text {coup }}\left(\boldsymbol{r}, \xi_{T}\right)$ is the potential for the coupling between the relative motion and the intrinsic motion of the target nucleus.

In the isocentrifugal approximation ${ }^{[1,33-35]}$, where the angular momentum of the relative motion in each channel is replaced with the total angular momentum $\mathrm{J}$, the coupled-channels equations derived from the Hamiltonian in Eq. (1) is obtained to be

$$
\begin{array}{r}
{\left[-\frac{\hbar^{2}}{2 \mu} \frac{d^{2}}{d r^{2}}+\frac{J(J+1) \hbar^{2}}{2 \mu r^{2}}+V_{N}^{0}(r)+\frac{Z_{P} Z_{T} e^{2}}{r^{2}}-E+\epsilon_{n}\right] u_{n}(r)} \\
+\sum_{n} V_{n n^{\prime}}(r) u_{n^{\prime}}(r)=0,
\end{array}
$$


where $\epsilon_{n}$ is the eigenvalue of the operator $H_{\text {exct }}$ for the $n$-th channel. $V_{n n^{\prime}}(r)$ is the matrix elements for the coupling potential $V_{\text {coup }}$.

In performing the coupled-channels calculations, we follow the method in Ref. [35] where the intrinsic coordinates, $\xi_{T}$, in the coupling potential, $V_{\text {coup }}$, is replaced with the dynamical excitation operator, $\hat{O}_{\lambda}$. The coupling potential is then represented by

$$
\begin{aligned}
& V_{\text {coup }}\left(r, \hat{O}_{\lambda_{T}}\right)=V_{C}\left(r, \hat{O}_{\lambda_{T}}\right)+V_{N}\left(r, \hat{O}_{\lambda_{T}}\right) \\
& V_{C}\left(r, \hat{O}_{\lambda_{T}}\right)=\frac{3 Z_{P} Z_{T} e^{2}}{\left(2 \lambda_{T}+1\right)} \frac{R_{T}^{\lambda_{T}}}{r^{\lambda_{T}+1}} \hat{O}_{\lambda_{T}} \\
& V_{N}\left(r, \hat{O}_{\lambda_{T}}\right)=\frac{-V_{0}}{\left\{1+\exp \left[\frac{r-R_{0}-R_{T} \hat{O}_{\lambda_{T}}}{a_{0}}\right]\right\}}+V_{N}^{0}(r)+\frac{-W_{0}}{\left\{1+\exp \left[\frac{r-R_{W}-R_{T} \hat{O}_{\lambda_{T}}}{a_{w}}\right]\right\}}
\end{aligned}
$$

where $R_{0}=r_{0}\left(A_{T}^{1 / 3}+A_{P}^{1 / 3}\right)$ and $R_{w}=r_{w}\left(A_{T}^{1 / 3}+A_{P}^{1 / 3}\right)$ with $A_{T}$ and $A_{P}$ are the mass number of the target and projectile nuclei, respectively. $\lambda_{T}$ denotes the multipolarity of the intrinsic excitation in the target nucleus. $R_{T}$ denotes the radius of the target nucleus and is taken to be $R_{T}=r_{\text {coup }} A_{T}^{1 / 3}$. We have subtracted $V_{N}^{0}(r)$ in Eq. (5) in order to avoid the double counting.

If we consider the spherical target nucleus, then the matrix element of the operator $\hat{O}_{\lambda_{T}}$ in Eqs. (3), (4), and (5) between the $n$-phonon states, $|n\rangle$, and the $m$-phonon state, $|n\rangle$, is given as ${ }^{[35]}$

$$
\hat{O}_{n m}=\frac{\beta_{\lambda_{T}}}{\sqrt{4 \pi}}\left(\sqrt{m} \delta_{n, m-1}+\sqrt{n} \delta_{n, m+1}\right)
$$

where $\beta_{\lambda_{T}}$ is the deformation parameter of the target nucleus. On the other hand, for deformed target nucleus, the coupling matrix element between the $|n\rangle=|I 0\rangle$ and $|n\rangle=\left|I^{\prime} 0\right\rangle$ states of the ground rotational band is given by ${ }^{[35]}$

$$
\hat{O}_{I I^{\prime}}=\sqrt{\frac{\left(2 \lambda_{T}+1\right)(2 I+1)\left(2 I I^{\prime}+1\right)}{4 \pi}} \beta_{\lambda_{T}}\left(\begin{array}{ccc}
I^{\prime} & \lambda_{T} & I \\
0 & 0 & 0
\end{array}\right) \text {. }
$$

The coupled-channels equations, Eq. (1), are solved with the scattering boundary condition for $u_{n}(r)$

$$
u_{n}(r) \rightarrow \frac{i}{2}\left[H_{J}^{(-)}\left(k_{n} r\right) \delta_{n, n_{i}}-\sqrt{\frac{k_{i}}{k_{n}}} S_{n}^{J} H_{J}^{(+)}\left(k_{n} r\right)\right] ; r \rightarrow \sim \infty
$$

where $S_{n}^{J}$ is the nuclear $S$-matrix. $H_{J}^{(-)}\left(k_{n} r\right)$ and $H_{J}^{(+)}\left(k_{n} r\right)$ are the incoming and the out going Coulomb wave functions, respectively. The channel wave number $k_{n}$ is given by $\sqrt{2 \pi\left(E-\epsilon_{n}\right) / \hbar^{2}}$ and $k_{i}=k_{n_{i}} \sqrt{2 \pi E / \hbar^{2}}$. The scattering angular distribution for the channel- $n$ is then given by ${ }^{[34]}$

$$
\frac{d \sigma_{n}}{d \Omega}=\frac{k_{n}}{k_{i}}\left|f_{n}(\theta)\right|^{2}
$$

with

$$
f_{n}(\theta)=\sum_{J} e^{\left[\sigma_{J}(E)+\sigma_{J}\left(E-\epsilon_{n}\right)\right]} \sqrt{\frac{(2 J+1)}{4 \pi}} Y_{J 0}(\theta) \frac{-2 i \pi}{\sqrt{k_{i} k_{n}}}\left(S_{n}^{J}-\delta_{n, n_{i}}\right)+f_{C}(\theta) \delta_{n, n_{i}}
$$


Here $\sigma_{J}(E)$ and $f_{C}(\theta)$ are the the Coulomb phase shift and the Coulomb scattering ampli-tude, respectively. The differential quasi-elastic cross section is then calculated to be

$$
\frac{d \sigma^{q e l}}{d \Omega}=\sum_{n} \frac{d \sigma_{n}}{d \Omega}
$$

And the corresponding fusion cross section is evaluated as

$$
\sigma_{f u s}=\frac{\pi}{k_{i}^{2}} \sum_{J}(2 J+1)\left(1-\left|S_{n}^{J}\right|^{2}\right.
$$

One will apply this formalism to perform the coupled-channels analysis for the fusion reaction and large quasi-elastic scattering of the ${ }^{16} \mathrm{O}+{ }^{144,154} \mathrm{Sm}$ systems.

\section{COUPLED-CHANNELS CALCULATIONS}

In this section, we present the results of our detailed coupled-channels analysis for fusion reaction and large angle quasi-elastic-scattering data of ${ }^{16} \mathrm{O}+{ }^{144,154} \mathrm{Sm}$ systems ${ }^{[10,14]}$. The calculations are performed using a computer code CQUEL ${ }^{[36]}$ which is a version of the coupled-channels code CCFULL ${ }^{[35]}$. In the code CQUEL, the regular boundary condition is employed at the origin, instead of the incoming boundary condition, and the restrictionof CCFULL which computes only the fusion cross sections is removed. Thus the code can calculate the fusion and quasielastic cross section simultaneously ${ }^{[37]}$. In the code, the isocentrifugal approximation employed. And it is found that the approximation is also works well for quasi-elastic scattering at backward angles ${ }^{[15]}$. A short range imaginary potential with $W_{0}=30 \mathrm{MeV}, r_{w}=1.0 \mathrm{fm}$, and $a_{w}=0.3 \mathrm{fm}$ is used to simulate the compound nucleus formation. The results of the fusion cross sections are insensitive to these parameters as long as the imaginary part of the potential is well confined inside the Coulomb barrier. All the calculations presented below are performed at the scattering angle of $\theta=170^{\circ}$. We plot the fusion cross sections and the fusion barrier distributions as a function of the center of mass energy, $E$. And the quasielastic cross sections and the quasi-elastic barrier distributions is plotted as a function of the effective energy defined by ${ }^{[14,15]}$

$$
E_{\text {eff }}=2 E \frac{\sin \left(\frac{\theta}{2}\right)}{1+\sin \left(\frac{\theta}{2}\right)}
$$

which takes into account the centrifugal energy. The fusion barrier distributions and the quasi-elastic barrier distributions are calculated in a similar way as those used to obtain the experimental barrier distributions ${ }^{[10,14]}$. Namely, we use point different formula with energy step $\Delta E=1.8 \mathrm{MeV}$ and $\Delta E=0.45 \mathrm{MeV}$ for calculating the fusion and the quasi- elastic barrier distributions, respectively. In the calculations presented below, we include only the excitations of ${ }^{144,154} \mathrm{Sm}$ nuclei whilst the excitations of the ${ }^{16} \mathrm{O}$ is not explicitly included. It has been shown in Ref. [38] that the later lead only to shift the fusion and the quasi-elastic barrier distributions in energy without altering its shape, and hence can be incorporated in the choice of the bare nuclear potential. 


\section{Effect Of Surface Diffuseness Parameter On The Fusion Reaction And Quasi-Elastic Scattering}

Let us first discuss the effect surface diffuseness parameter of the nuclear potential on the fusion and quasi-elastic barrier distribution for ${ }^{16} \mathrm{O}+{ }^{144,154} \mathrm{Sm}$ systems. We use the surface diffuseness parameter of the nuclear potential $a=0.63 \mathrm{fm}, a=0.75 \mathrm{fm}$, , and $a=0.87 \mathrm{fm}$, respectively for the ${ }^{16} \mathrm{O}+{ }^{144} \mathrm{Sm}$ system. These values have been used to explain the experimental fusion data ${ }^{[10,38,39]}$ and quasi-elastic scattering data of this system ${ }^{[7,8,39]}$. On the other hand, for the ${ }^{16} \mathrm{O}+{ }^{154} \mathrm{Sm}$ system, we used $a=$ $0.63 \mathrm{fm}$ and $a=1.05 \mathrm{fm}$. The value of $a=0.63 \mathrm{fm}$ was found to well describes the experimental quasi-elastic scattering data ${ }^{[7,9,28-31]}$. And the value of $a=$ $1.05 \mathrm{fm}$ was required by the experimental fusion data ${ }^{[10]}$. The depth, $V_{0}$, and the radius, $r_{0}$, parameters for each surface diffuseness parameter are adjusted in order to reproduce the same Coulomb barrier height for each system. All these parameters are tabulated in Table I.

Table I. The parameters of the nuclear potential for the ${ }^{16} \mathrm{O}+{ }^{144,154} \mathrm{Sm}$ systems used in the coupledchannels calculations. The resultant Coulomb barrier height $\mathrm{V}_{B}$ in $\mathrm{MeV}$ is also listed.

\begin{tabular}{rcccc}
\hline System & $V_{0}(\mathrm{MeV})$ & $a_{0}(\mathrm{fm})$ & $a(\mathrm{fm})$ & $V_{B}(\mathrm{MeV})$ \\
\hline${ }^{16} \mathrm{O}+{ }^{144} \mathrm{Sm}$ & 91.70 & 1.16 & 0.63 & 61.25 \\
& 105.10 & 1.10 & 0.75 & 61.25 \\
& 167.20 & 1.00 & 0.87 & 61.25 \\
\hline${ }^{16} \mathrm{O}+{ }^{154} \mathrm{Sm}$ & 95.30 & 1.18 & 0.63 & 59.41 \\
\hline
\end{tabular}

Figure 1 shows the results of coupled-channels calculations for the ${ }^{16} \mathrm{O}+{ }^{144} \mathrm{Sm}$ system using three different values of the surface diffuseness parameters of the nuclear potential. We include the quadrupole $\left(2^{+}\right)$and the octupole $\left(3^{-}\right)$excitations in the target nucleus whose excitation energies are given as $\epsilon_{2}=1.66 \mathrm{MeV}$ and $\epsilon_{3}=1.81 \mathrm{MeV}$, respectively. The associated deformation parameters are $\beta_{2}=0.112$

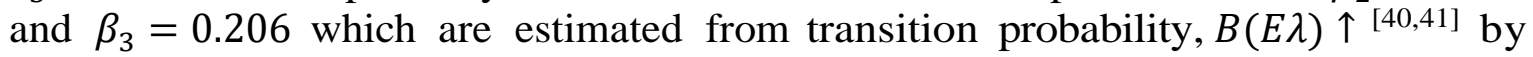
using $r_{\text {coup }}=1.06 \mathrm{fm}$. Figs. 1(a) and 1(b) show the fusion cross section and the fusion barrier distribution. The quasi-elastic cross section and the corresponding barrier distribution are given in Figs. 1(c) and 1(d). The dashed and the solid lines are the results of the calculations using $a=0.63 \mathrm{fm}$ and $a=0.75 \mathrm{fm}$, respectively. And the dotted line is the results using $a=0.87 \mathrm{fm}$. We observe that the calculated fusion cross section and fusion barrier distribution well reproduced the experimental data. The structure of the barrier distributions obtained with the three different surface diffuseness parameters are similar to each other. However, the obtained fusion barrier using small surface diffuseness parameter has a smoother high energy peak compared to others results. The similar situation are also found for the quasi-elastic barrier distribution. It is found that the calculated quasi-elastic barrier distribution using $a=0.87 \mathrm{fm}$ underestimated the experimental data. The experimental quasi-elastic cross sections require the small value for the surface diffuseness parameter as those previously found in many 
studies ${ }^{[9,28-31]}$. These results indicate that the structure of the fusion and the quasi-elastic barrier distributions are sensitive to the surface diffuseness parameter where the small diffuseness parameter seem to smeared-out the barrier distributions.
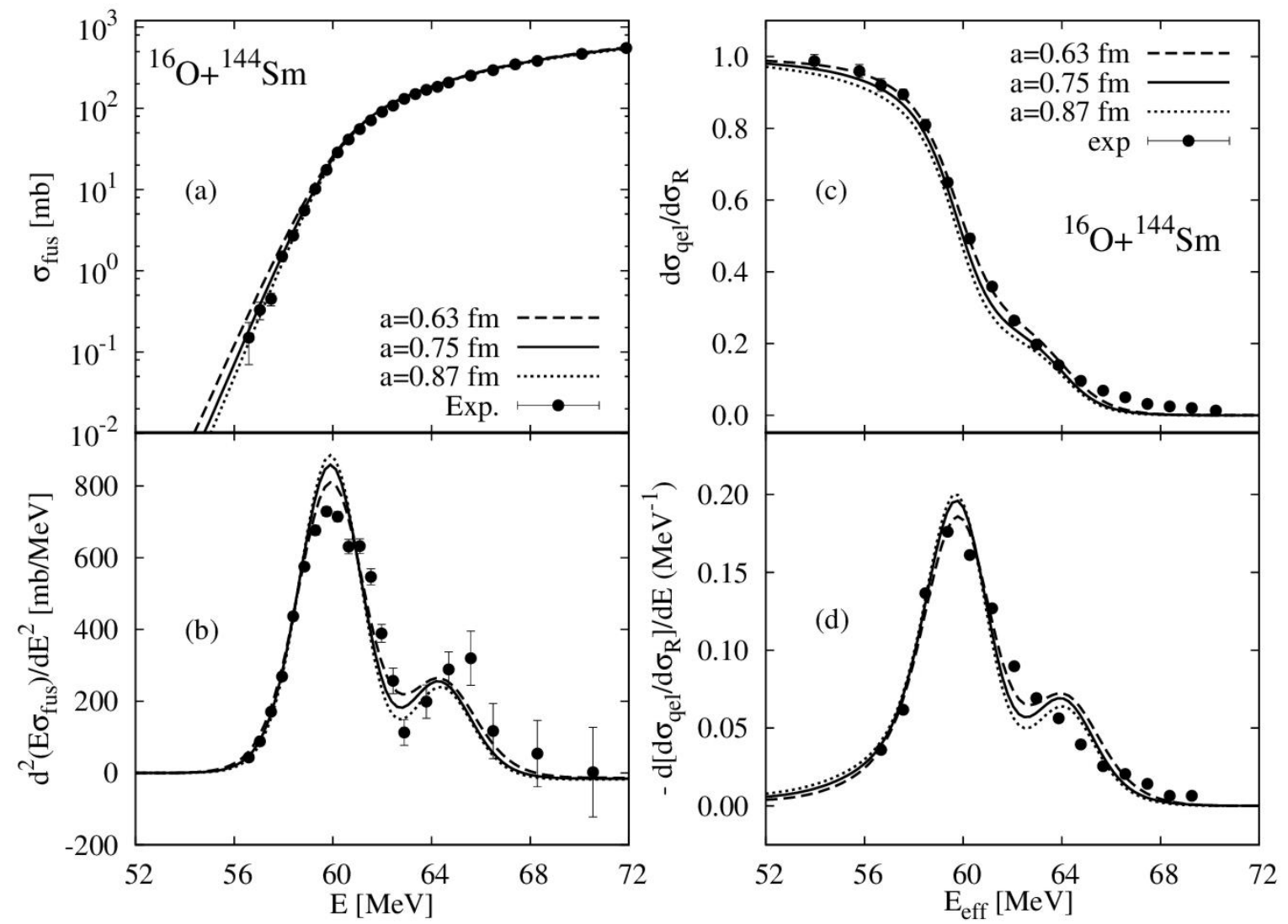

Figure 1. Comparison of (a) the excitation function of the fusion cross section, (b) the fusion barrier distribution,

(c) the quasi-elastic cross section, and (d) the quasi-elastic barrier distribution for the ${ }^{16} \mathrm{O}+{ }^{144} \mathrm{Sm}$ reaction obtained with three different values of the surface diffuseness parameter. The dashed and the solid lines are the results of coupled-channels calculations by using $a=0.63 \mathrm{fm}$ and $a=0.75 \mathrm{fm}$, respectively. And the dotted line is obtained with $a=0.87 \mathrm{fm}$. Experimental data are taken from Refs. ${ }^{[10,14]}$.

We now discuss the coupled-channels calculations for the ${ }^{16} \mathrm{O}+{ }^{154} \mathrm{Sm}$ system. We use the deformation parameters $\beta_{2}=0.31$ and $\beta_{4}=0.05$ with $r_{\text {coup }}=1.06 \mathrm{fm}$ for the target nucleus ${ }^{[15,32]}$. The ground states of the rotational bands are included up to $10^{+}$members. The results are given in Figs. 2(a) and 2(b) for the fusion cross section and the fusion barrier distribution, respectively. The quasi-elastic cross section and the quasi-elastic barrier distribution are presented in Figs. 2(c) and 2(d), respectively. The dashed line denotes the results of the calculations using $a=$ $0.63 \mathrm{fm}$ whereas the solid lines using $a=1.05 \mathrm{fm}$. The obtained fusion cross section and fusion barrier distribution with these two different values of the surface diffuseness parameters are similar to each other and they well describe the experimental data. Though the calculated quasi-elastic cross section using a different values for surface diffuseness parameter are disagree to each other where the calculated quasi-elastic scattering with $a=1.05$ well explain the data especially in the energies below the Coulomb barrier (noticed that this phenomena has been already clarified in Ref. [29]) but the structure of the resultant quasielastic barrier distributions are similar to each other. Thus it is obvious that the 
fusion and quasi-elastic barrier distributions for the ${ }^{16} \mathrm{O}+{ }^{154} \mathrm{Sm}$ systems are less sensitive to the surface diffuseness parameter of the nuclear potential.
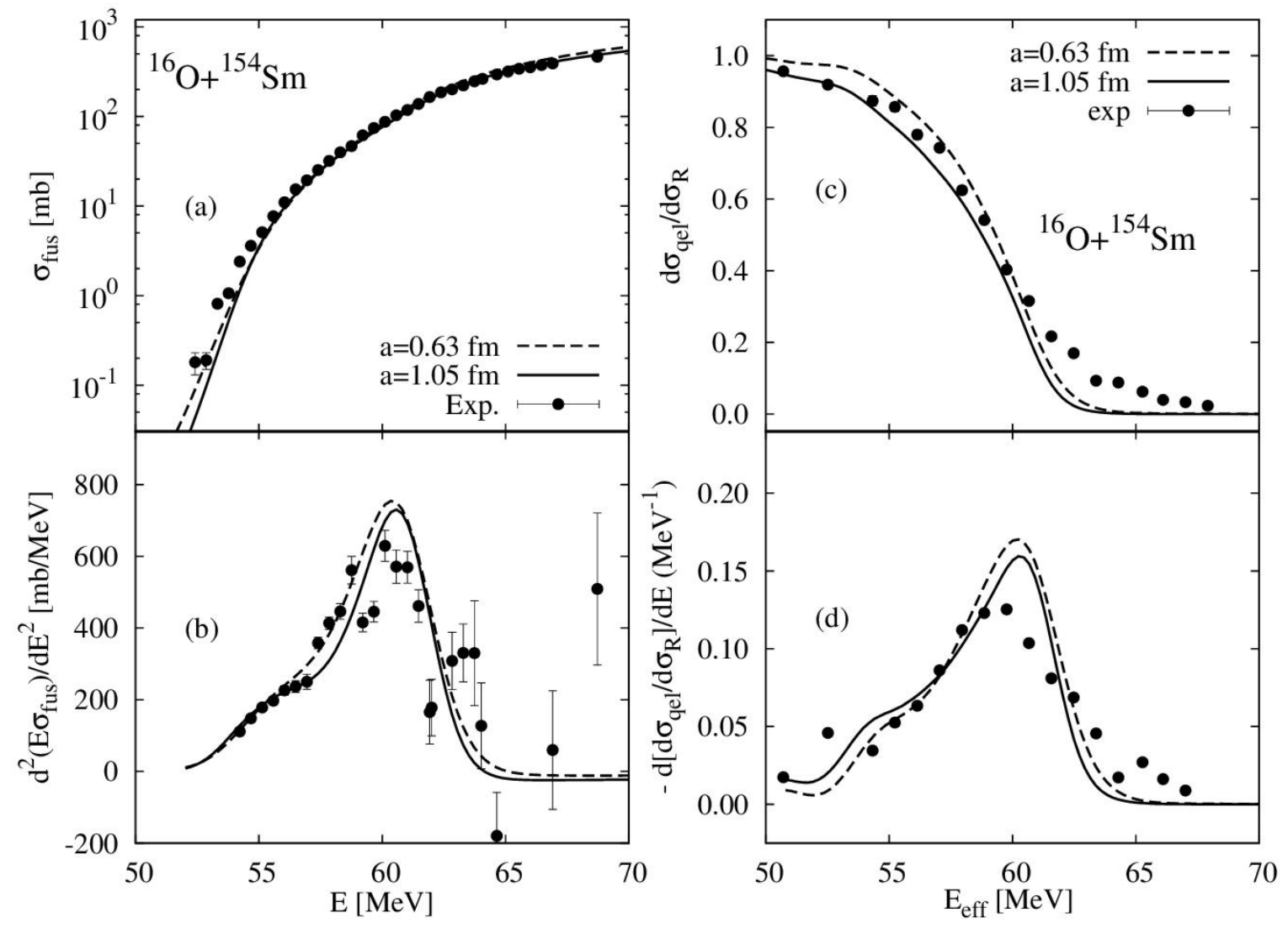

Figure 2. The same as Figure 1 but for ${ }^{16} \mathrm{O}+{ }^{154} \mathrm{Sm}$ system. The solid and dashed lines are obtained by using $a=1.05 \mathrm{fm}$ and $a=0.63 \mathrm{fm}$, respectively. Experimental data are taken from Refs. [10, 14].

\section{Effect Of Surface Diffuseness Parameter On The Fusion Reaction And Quasi-Elastic Scattering}

In this subsection, we investigate the sensitivity of coupled-channels calculations for the fusion and quasi-elastic scattering of ${ }^{16} \mathrm{O}+{ }^{144,154} \mathrm{Sm}$ systems to the coupling radius parameter. We use two values of the coupling radius parameter that is $r_{\text {coup }}=$ $1.06 \mathrm{fm}$ as used in the previous subsections and $r_{\text {coup }}=1.20 \mathrm{fm}$ as that use in Ref. [9] when they performed the coupled-channels calculations for large angle quasi-elastics in massive systems. The estimated deformation parameters with this coupling radius parameter are $\beta_{2}=0.10$ and $\beta_{3}=0.142$ for the quadrupole and octupole excitations, respectively, in ${ }^{144} \mathrm{Sm}$ nucleus. For the ${ }^{154} \mathrm{Sm}$ nucleus, the corresponding deformation parameters are found to be $\beta_{2}=0.274$ and $\beta_{4}=$ 0.044. In performing the coupled-channels calculations, the surface diffuseness of $a=$ $0.75 \mathrm{fm}$ is used for ${ }^{16} \mathrm{O}+{ }^{144} \mathrm{Sm}$ reaction since the calculation are well reproduced the experimental data as shown in the previous subsection and for ${ }^{16} \mathrm{O}+{ }^{154} \mathrm{Sm}$, we use $a=$ $1.05 \mathrm{fm}$. The procedure of the calculations is the same as the previous subsection. 

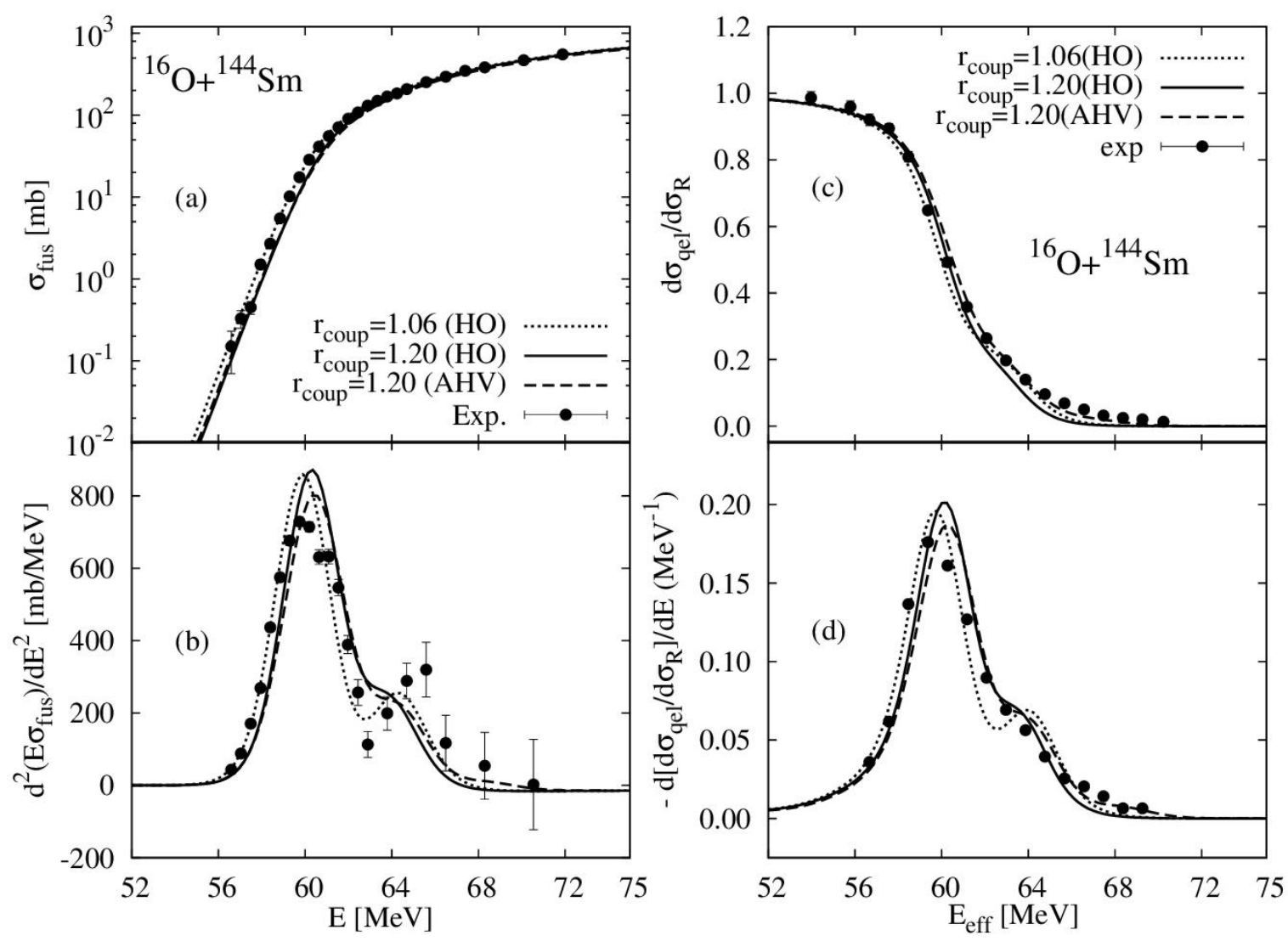

Figure 3. Sensitivity of (a) the fusion excitation function, (b) the fusion barrier distribution, (c) the quasielastic cross section, and $(\mathrm{d})$ the quasi-elastic barrier distribution on the coupling radius parameter for ${ }^{16} \mathrm{O}+{ }^{144} \mathrm{Sm}$ system. The dashed line is the result of the coupled-channels calculations by using $r_{\text {coup }}=1.06 \mathrm{fm}$ while the solid line by using $r_{\text {coup }}=1.20 \mathrm{fm}$. Experimental data are taken from Refs. [10, 14].

The results of the coupled-channels calculations with different coupling radius parameter is presented in Fig. 3 for ${ }^{16} \mathrm{O}+{ }^{144} \mathrm{Sm}$ system. Figures 3(a) and 3(b) show the fusion cross section and the fusion barrier distribution, respectively. And Figures 3(c) and 3(d) represent the quasi-elastic cross section and the quasi-elastic barrier distributions, respectively. The dotted lines are the results using the coupling radius, $r_{\text {coup }}=1.06 \mathrm{fm}$, while the solid lines are obtained by using the coupling radius $r_{\text {coup }}=1.20 \mathrm{fm}$. For comparison, we also show the results of the calculations by taking into account the anharmonic of the vibration excitations, as those used in Ref. [8], with coupling radius $r_{\text {coup }}=1.20 \mathrm{fm}$ (in this case, the deformation parameters for quadrupole and octupole excitation are $\beta_{2}=0.21$ and $\beta_{3}=0.09$, respectively). It is observed that the experimental data of fusion cross section and the quasi- elastic cross section are well reproduced. The fusion barrier distribution obtained with $r_{\text {coup }}=1.06 \mathrm{fm}$ is more well agree with the experimental data compared the one obtained using $r_{\text {coup }}=1.20 \mathrm{fm}$. On the other hand, the quasielastic barrier distribution are well explained with the calculations using the larger coupling radius, i.e. $r_{\text {coup }}=1.20 \mathrm{fm}$. The reason for discrepancy of the value of the coupling radius parameter used in the calculations for fusion and quasi-elastic scattering has not yet understood by now and it is still an open problem. 

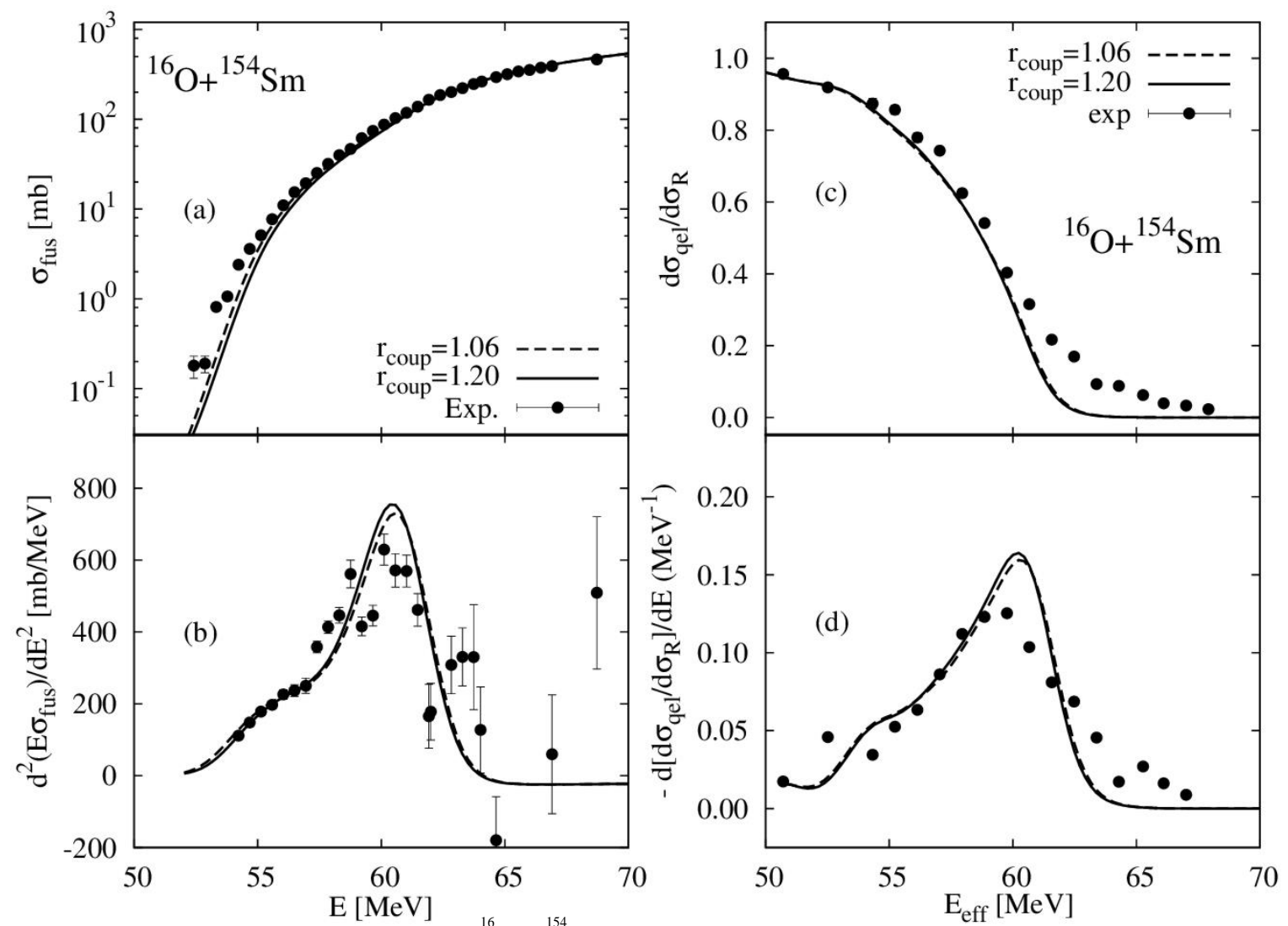

Figure 4. The same as Figure. 3 but for ${ }^{16} \mathrm{O}+{ }^{154} \mathrm{Sm}$ system. Experimental data are taken from Refs. [10, 14].

The results of the coupled-channels calculations for ${ }^{16} \mathrm{O}+{ }^{154} \mathrm{Sm}$ system using different coupling radius parameters is shown in Fig. 4. Figures 4(a) and 4(c) show the fusion and quasi-elastic cross section, respectively. And Figures 4(b) and 4(d) represent the fusion and quasi-elastic barrier distributions, respectively. The dotted lines are the results using the coupling radius, $r_{\text {coup }}=1.06 \mathrm{fm}$, while the solid lines are obtained by using the coupling radius $r_{\text {coup }}=1.20 \mathrm{fm}$. The calculated fusion and quasi-elastic cross sections obtained with these two values of coupling radius are similar to each other and they well reproduced the experimental data. The similar situation is also found for the barrier distributions. It indicates that the coupling radius parameters are not affect the structure of the fusion and the quasi-elastic barrier distributions of this system.

\section{CONCLUSIONS}

We have performed the coupled-channels calculations for fusion reaction and quasielastic scattering for ${ }^{16} \mathrm{O}+{ }^{144,154} \mathrm{Sm}$ systems. We especially analyze the effect of the surface diffuseness and the coupling radius parameters of the coupling potential on the fusion and quasi- elastic barrier distributions. It is found that for reaction with deformed target, ${ }^{16} \mathrm{O}+{ }^{154} \mathrm{Sm}$ system, the structure of fusion and quasi-elastic barrier distributions is not sensitive to the choice of the surface diffuseness and the coupling radius parameters. However, the fusion and quasi-elastic barrier distribution for reaction that involves the spherical target, ${ }^{16} \mathrm{O}+{ }^{144} \mathrm{Sm}$ system, are sensitive to the surface diffuseness and the coupling radius parameters. The calculated fusion and quasi-elastic barrier distributions with small diffuseness parameter have a more smooth high energy peak. An interesting fact is found, i.e. the coupled-channels 
calculations of the fusion barrier distributions seems to require the small coupling radius, $r_{\text {coup }}=1.06 \mathrm{fm}$ in order to explain the experimental data. On the other hand, the quasi-elastic barrier distributions require the 1 a rge $\mathrm{r}$ coupling radius, $r_{\text {coup }}=$ $1.20 \mathrm{fm}$, for fitting the data. With this finding, the further study is still necessary in order to clarify this discrepancy. Its still an open problem to carry out the coupled-channels calculations by considering another effect such as dynamical effects for explaining the difference of shape between the fusion and the quasi-elastic barrier distributions for ${ }^{16} \mathrm{O}+{ }^{144} \mathrm{Sm}$ system.

\section{ACKNOWLEDGMENTS}

This work is supported by Fundamental Research Grant from Ministry of Research, Technology and Higher Education of the Republic of Indonesia.

\section{REFERENCES}

1 Balantekin, A. B. \& Takigawa, N., 1998, Quantum Tunneling In Nuclear Fusion, Rev. Mod. Phys., 70 (77).

2 Dasgupta, M., Hinde, D. J., Rowley, N., \& Stefanini, A. M., 1998, Measuring Barriers To Fusion, Annu. Rev. Nucl. Part. Sci., 48 (401).

3 Canto, L. F., Gomes,P. R. S., Donangelo, R., \& Hussein, M. S., 2006, Fusion And Breakup Of Weakly Bound Nuclei, Phys. Rep., 424 (1).

4 Keeley, K., Raabe, R., Alamanos, N., \& Sida, J. L., 2007, Fusion and Direct Reactions Of Halo Nuclei At Energies Around The Coulomb Barrier, Prog. Part. Nucl. Phys. 59 (579).

5 Back, B. B., Esbensen, H., Jiang, C. L., \& Rehm, K. E., 2014, Recent Developments In Heavy-Ion Fusion Reactions, Rev. Mod. Phys., 86 (317).

6 Hagino, K. \& Takigawa, N., 2012, Subbarrier Fusion Reactions and Many-Particle Quantum Tunneling, Prog. Theo. Phys., 128 (1061).

7 Muhammad Zamrun, F., 2016, Role Of Triple Phonon Excitations In Large Angle Quasi-Elastic Scattering Of Very Heavy Mass Systems, Int. J. Mod. Phys. E, 25 (1650054).

8 Muhammad Zamrun, F. \& Hagino, K., 2008, Effects Of Anharmonic Vibration On Large-Angle Quasi-Elastic Scattering Of ${ }^{16} \mathrm{O}+{ }^{144} \mathrm{Sm}$, Phys. Rev. C, 77 (014606).

9 Muhammad Zamrun, F., Hagino, K., Mitsuoaka, S., \& Ikezoe, H., 2008 , Coupled-Channels Analyses For Large-Angle Quasi-Elastic Scattering In Massive Systems, Phys. Rev. C, 77 (034604).

10 Leigh, J. R., Dasgupta, M., Hinde, D. J., Mein, J. C., Morton, C. R., Lestone, J. P., Newton, J. O., Timmers, H., Wei, J. X., \& Rowley, N., 1995, Barrier Distributions From The Fusion Of Oxygen Ions With ${ }^{144,148,154}$ Sm And ${ }^{186} \mathrm{~W}$, Phys. Rev. C, 52 (3151).

11 Rowley, N., Grar, N., \& Hagino, K., 2006, An extra push from entrance-channel effects, Phys. Lett. B, 632 (243).

12 Zamrun, M., Usman, I., Variani, V. I., \& Kassim, H. A., 2014, Sensitivity Of Fusion And Quasi-Elastic Barrier Distributions Of ${ }^{16} \mathrm{O}+{ }^{144} \mathrm{Sm}$ Reaction On The Coupling Radius Parameter, AIP Conf. Proc., 1588 (221).

13 Rowley, N., Sacthler, G. R., \& Stelson, P. H., 1991, On The "Distribution Of Barriers" Interpretation Of Heavy-Ion Fusion, Phys. Lett. B, 254 (25).

14 Timmers, H., Leigh, J. R., Dasgupta, M., Hinde, D. J., Lemon, R. C., Mein, J. C., Morton, C. R., Newton, J. O., \& Rowley, N., 1995, Probing Fusion Barrier Distributions With Quasi-Elastic Scattering, Nucl. Phys. B, 584 (190). 
15 Hagino, K. \& Rowley, N., 2004, Large-Angle Scattering and Quasielastic Barrier Distributions, Phys. Rev. C, 69 (054610).

16 Andres, M. V., Rowley, N., \& Nagarajan, M. A., 1988, Effect Of Deformation On The Elastic And Quasielastic Scattering Of Heavy Ions Near The Coulomb Barrier, Phys. Lett. B, 202 (292).

17 Rowley, N., et al., 2006, Capture Cross Sections for Very Heavy Systems, Phys. At. Nucl.. 69 (1093).

18 Ntshangase, S. S., et al., 2007, Barrier Distribution for a 'Superheavy' NucleusNucleus Collision, Phys. Lett. B, 651 (27).

19 Ikezoe, H., Mitsuoka, S., Nishio, K., Watanabe, Y., Jeong, S.C., Nishinaka, I., Ohtsuki, T., \& Hirose, J., 2010, Dependence of Barrier Distribution and FusionFission Process on Entrance Channel, Nucl. Phys. A, 834 (172c).

20 Ikezoe, H., Mitsuoka, S., Nishio, K., Tsuruta, K., Watanabe, Y., Jeong, S. C., \& Satou, K., 2006, Barrier Distributions for Cold-Fusion Reactions, AIP Conf. Proc., 853 (69).

21 Mitsuoka, S., Ikezoe, H., Nishio, K., Tsuruta, K., Jeong, S. C., \& Watanabe, Y., 2007, Barrier Distributions Derived from Quasielastic Backscattering of ${ }^{48} \mathrm{Ti},{ }^{54} \mathrm{Cr}$, ${ }^{56} \mathrm{Fe},{ }^{64} \mathrm{Ni}$, and ${ }^{70} \mathrm{Zn}$ Projectiles on a ${ }^{208} \mathrm{~Pb}$ Target, Phys. Rev. Lett., 99 (182701).

22 Mukherjee, A., Hinde, D. J., Dasgupta, M., Hagino, K., Newton, J. O., \& Butt, R. D., 2007, Failure of the Woods-Saxon nuclear potential to simultaneously reproduce precise fusion and elastic scattering measurements, Phys. Rev. C, 75 (044608).

23 Gontchar, I. I., Hinde, D. J., Dasgupta, M., \& Newton, J. O., 2004, Double folding nucleus-nucleus potential applied to heavy-ion fusion reactions, Phys. Rev. C, 69 (024610).

24 Hagino, K., Rowley, N., \& Dasgupta, M, 2003, Fusion cross sections at deep subbarrier energies, Phys. Rev. C, 67 (054603).

25 Newton, J. O., Morton, C. R., Dasgupta, M., Leigh, J. R., Mein, J. C., Hinde, D. J., Timmers, H., \& Hagino, K., 2001, Experimental barrier distributions for the fusion of ${ }^{12} \mathrm{C},{ }^{16} \mathrm{O},{ }^{28} \mathrm{Si}$, and ${ }^{35} \mathrm{Cl}$ with ${ }^{92} \mathrm{Zr}$ and coupled-channels analyses, Phys. Rev. C, 64 (064608).

26 Newton, J. O., Butt, R. D., Dasgupta, M., Hinde, D. J., Gontchar, I. I., Morton, C. R., \& Hagino, K., 2004, Systematic failure of the Woods-Saxon nuclear potential to describe both fusion and elastic scattering: Possible need for a new dynamical approach to fusion, Phys. Rev. C, 70 (024605).

27 Ibrahim, M. L. I., Zamrun, M., \& Kassim, H. A., 2013, Analysis of the nuclear potential for heavy-ion systems through large-angle quasi-elastic scattering, Phys. Rev. $C, 87$ (024611).

28 Evers, M., Dasgupta, M., Hinde, D. J., Gasques, L. R., Brown, M. L., Rafiei, R., \& Thomas, R. G., 2008, Systematic study of the nuclear potential diffuseness through high precision back-angle quasi-elastic scattering, Phys. Rev. C, 78 (034614).

29 Gasques, L. R., Evers, M., Hinde, D. J., Dasgupta, M., Gomes, P. R. S., Anjos, R. M., Brown, M. L., Rodriguez, M. D., Thomas, R. G., \& Hagino, K., 2007 , Systematic study of the nuclear potential through high precision back-angle quasi-elastic scattering measurements, Phys. Rev. C, 76 (024612).

30 Hinde, D. J., Ahlefeldt, R. L., Thomas, R. G., Hagino, K., Brown, M.L., Dasgupta, M., Evers, M., Gasques, L. R., \& Rodriguez, M. D., 2007 , Probing the tail of the nuclear potential between identical nuclei with quasi-elastic Mott scattering, Phys. Rev. C 76, 014617 (2007). 
31 Washiyama, K., Hagino, K., \& Dasgupta, M., 2006, Probing surface diffuseness of nucleus-nucleus potential with quasielastic scattering at deep sub-barrier energies, Phys. Rev. C, 73 (034607).

32 Hagino, K., Takehi, T., Balantekin, A. B., \& Takigawa, N., 2005, Surface diffuseness anomaly in heavy-ion potentials for large-angle quasielastic scattering, Phys. Rev. C, 71 (044612).

33 Takigawa, N. \& Ikeda, K., 1986, ANL-PHY-86-1, Proc. of the Intl. Symposium on the Many Facets of Heavy Ion Fusion Reactions, Argone National Laboratory, 613-620.

34 Esbensen, H., Landowne, S., \& Price, C., 1987, Comment on "Reduction of coupled equations for heavy ion reactions', Phys. Rev. C, 36 (1216); High-spin excitations in the rotating frame and sudden approximations, 1987, Phys. Rev. C, 36 (2359).

35 Hagino, K., Rowley, N., \& Kruppa, A. T., 1999, A program for coupled-channel calculations with all order couplings for heavy-ion fusion reactions, Comput. Phys. Commun., 123 (143).

36 K. Hagino et al. (to be published).

37 Muhammad Zamrun, F., \& Hagino, K., 2009, Role of Anharmonic Vibration on Heavy-ion Fusion Reaction and Large Angle Quasi-elastic Scattering of ${ }^{16} \mathrm{O}+{ }^{144} \mathrm{Sm}$, AIP Conf. Proc., 1150 (464).

38 Hagino, K., Takigawa, N., \& Kuyucak, S., 1997, Role of Anharmonicities of Nuclear Vibrations in Fusion Reactions at Sub-barrier Energies, Phys. Rev. Lett., 79 (2943).

39 Esbensen, H. \& Back, B. B., 1996, Coupled channels analysis of high precision fusion data, Phys. Rev. C, 54 (3109).

40 Kibedi, T. \& Spears, R. H., 2002, Reduced Electric-Octupole Transition Probabilities, $B\left(E 3 ; 0_{1}{ }^{+} \rightarrow 31^{-}\right)$-An Update, At. Data Nucl. Data Tables, 80 (35).

41 Raman, S., Nestor, C. W., \& Tikkanen, P., 2001, Transition Probability From The Ground To The First-Excited $2^{+}$State Of Even-Even Nuclides, At. Data Nucl. Data Tables, 78 (1). 